1990 ) and $6 \%$ of patients have been reported to develop tricyclic-induced delirium on conventional doses of $100-300 \mathrm{mg} /$ day. At plasma levels greater than $1000 \mu \mathrm{g} / 1$, nearly all will show ECG changes (Spiker et al, 1975). The first sign of cardiac toxicity may be a fatal arrhythmia.

Optimisation of clinical response is only one goal of therapeutic drug monitoring and even when well-defined plasma levels are not established for certain tricyclics, it still remains a useful tool in the management of depression. By detecting asymptomatic toxicity, therapeutic drug monitoring can prevent adverse consequences and may have medicolegal implications. Other advantages include assessment of nonresponders, provision of a measure of compliance and confirmation of toxicity. In contrast to Taylor and Duncan, we believe that routine estimation of plasma tricyclic levels is an integral component in the rational approach to the management of depression and should be more widely practised.

PRESKORN, S. H. (1993) Sudden death and tricyclic antidepressants (TCAs): a rare adverse event linked to high TCA plasma levels. Nordic Journal of Psychiatry, 47. (Suppl. 30), 49-55.

- JERKOVICH, G. S. (1990) Central nervous system toxicity of tricyclic antidepressants: phenomenology, course, risk factors and role of therapeutic drug monitoring. Journal of Clinical Psychopharmacology, 10, 88-95.

SPIKER, D. G., Weiss, A. N., Chang, S. S. (1975) Tricyclic antidepressant overdose: clinical presentation and plasma levels. Clinical Pharmacology and Therapeutics, $18,539-546$.

P. A. Cotter and P. W. RAVEN

Section of Metabolic Studies,

Institute of Psychiatry, London SE5 8AF

Sir: Toxicity may indeed occur in the absence of clinical symptoms. However, 'CNS toxicity' and delirium are clinical symptoms and so plasma level determinations are of little use apart from to confirm that the antidepressant is the cause of the problem. Electrocardiogram (ECG) abnormalities may be asymptomatic and so a plasma level may help identify those at risk. However, an ECG will still need to be performed to identify any arrhythmia and one might argue that this should be done for anyone taking moderate or high doses of tricyclics. We are also unsure of the value of a cut-off level of $1000 \mathrm{mcg} / \mathrm{l}$. Presumably anyone with a level above this would have an ECG performed. One wonders what course of action would be taken with a patient with a level of $999 \mathrm{mcg} / \mathrm{l}$, or $950 \mathrm{mcg} / 1$, or $800 \mathrm{mcg} / \mathrm{l}$. We feel the quickest way to detect occult rhythm abnormalities in patients taking tricyclic and related antidepressants is immediately to perform an ECG.

D. Duncan and D. TAYLOR

The Maudsley Hospital, London SE5 8AZ

\section{Improper terminology}

Sir: We warmly welcome the issues raised in the paper by Haghighat \& Littlewood (Psychiatric Bulletin, July 1995, 19, 407-410) which raises the issue of potential labelling and stigmatisation of people suffering from mental disorders. It is our duty to treat people with respect; as individuals, yet holistically. This should be made clear in the way that we, as professionals, refer to patients, their problems, and their illnesses.

Since Haghighat \& Littlewood's paper, we have been surprised and disappointed to note the continued use of terms such as 'schizophrenics' (e.g. Fagin et al, Psychiatric Bulletin, August 1995, 19, 533) and even 'dements' (Psychiatric Bulletin. November 1995, 19, 704) to refer to patients.

We strongly believe that as "The Journal of trends in psychiatric practice" the Bulletin should take the moral lead on this issue, and avoid publication of such pejorative and stigmatising labels.

David LaWley, MatTheW MaRChant and JOHN BESTLEY De La Pole Hospital, Willerby, Hull HU10 6ED

Point taken. - Ed.

\section{Correcting drug-induced hyperprolactinaemia}

Sir: Duncan and Taylor (Psychiatric Bulletin, December 1995, 19, 755-757) describe possible clinical usage of two drugs relatively unfamiliar to psychiatrists, amantadine and bromocriptine. They suggest this would correct a common sideeffect of antipsychotic drugs, hyperprolactinaemia. We believe that a simpler strategy should be followed initially.

Patients treated with antipsychotic medication can experience a variety of unpleasant endocrine side-effects; most commonly gynaecomastia, galactorrhoea and amenorrhoea. This is considered to be due to hyperprolactinaemia caused by antagonism of the action of dopamine on tuberoinfundibular neurones (Meltzer \& Fang, 1976).

The atypical neuroleptic clozapine is known to cause either a minimal or no rise in serum prolactin (Jann et al, 1993). Clozapine is indicated for the treatment of schizophrenia in patients unresponsive to, or intolerant of, conventional antipsychotic drugs (British National Formulary, 1995).

Our practice, in this not uncommon clinical situation, would be to change to clozapine. Duncan and Taylor point out that amantadine may precipitate mania and is unlicensed for hyperprolactinaemia. They also point out that bromocriptine is contraindicated in any psychotic 